\title{
Reductive decompositions and Einstein-Yang-Mills equations associated to the oscillator group
}

\author{
Raúl Durán Díaz \\ Instituto de Física Aplicada, CSIC, \\ Serrano 144, 28006-Madrid, Spain \\ Pedro M. Gadea \\ Instituto de Matemáticas y Física Fundamental, CSIC, \\ Serrano 123, 28006-Madrid, Spain \\ José A. Oubiña \\ Departamento de Xeometría e Topoloxía, Facultade de Matemáticas, \\ Universidade de Santiago de Compostela, 15706-Santiago de Compostela, Spain
}

Short title: Oscillator group

PACS numbers: 04.40.Nr, 11.15.-q, 02.40.Ma

M.S. Classification (1991): 53C30, 53C50, 53C80

\begin{abstract}
All of the homogeneous Lorentzian structures on the oscillator group equipped with a biinvariant Lorentzian metric, and then the associated reductive pairs, are obtained. Some of them are solutions of the Einstein-Yang-Mills equations.
\end{abstract}




\section{INTRODUCTION}

The oscillator group has interesting features from the viewpoints of both Differential Geometry and Physics (see for instance Refs. [1]-[12]). It was introduced by Streater [1], who named it so because its Lie algebra can be identified to that generated by the differential operators associated to the harmonic oscillator problem, acting on functions of a variable. The oscillator group is the only simply connected 4-dimensional nonabelian solvable Lie group which admits a biinvariant Lorentzian metric [3, 4]. Moreover, it is [8] an example of homogeneous spacetime, which as causal space satisfies the so-called causal continuity.

Levichev studied in Ref. [5] the oscillator group with the biinvariant Lorentzian metric also given in Refs. [6] and [8], proving that this group (which geometrically is a Lorentzian symmetric space and phisically is related to an isotropic electromagnetic field) provides a solution of the sourceless Einstein-Yang-Mills equations.

On the other hand, generalizing the classical characterization by É. Cartan [13] of Riemannian symmetric spaces as the spaces of parallel curvature, Ambrose and Singer [14] gave a characterization of Riemannian homogeneous spaces in terms of a $(1,2)$ tensor field $S$, called homogeneous Riemannian structure in Ref. [15], satisfying certain differential equations (see (1) below). In Refs. [16] and [17], we have extended this concept to the pseudo-Riemannian case.

In Ref. [12], all of the homogeneous Lorentzian structures corresponding to a family of left invariant metrics on the general oscillator groups have been obtained, and the reductive decompositions for the non-biinvariant metrics in that family have been determined.

In this paper we consider the 4-dimensional oscillator group equipped with its usual Lorentzian biinvariant metric and we determine all the homogeneous Lorentzian structures on it and all the associated reductive pairs. There appear six types of such pairs, and we prove that four of them are solutions of the Einstein-Yang-Mills equations. They have sources except for a particular case, where the symmetric pair appears as a solution of the sourceless Einstein-Yang-Mills equations.

\section{PRELIMINARIES}

Ambrose and Singer [14] gave a characterization for a connected, simply connected and complete Riemannian manifold to be homogeneous, in terms of a $(1,2)$ tensor field $S$, called in Ref. [15] a homogeneous Riemannian structure. In Ref. [16] it is defined a homogeneous pseudo-Riemannian structure on a pseudo-Riemannian manifold $(M, g)$ as a tensor field $S$ of type $(1,2)$ such that being $\nabla$ the Levi-Civita connection and $R$ its curvature tensor, the connection $\widetilde{\nabla}=\nabla-S$ satisfies the Ambrose-Singer equations

$$
\widetilde{\nabla} g=0, \quad \widetilde{\nabla} R=0, \quad \widetilde{\nabla} S=0 .
$$

In Ref. [16] it is proved that if $(M, g)$ is connected, simply connected and geodesically complete then it admits a homogeneous pseudo-Riemannian structure if and only if it is a reductive homogeneous pseudo-Riemannian manifold, which means that $M=G / H$, where $G$ is a connected Lie group acting transitively and effectively on $M$ as a group of isometries, $H$ is the isotropy group at a point $o \in M$, and the Lie algebra $\mathfrak{g}$ of $G$ may be decomposed into 
a vector space direct sum of the Lie algebra $\mathfrak{h}$ oh $H$ and an $\operatorname{Ad}(H)$-invariant subspace $\mathfrak{m}$, that is $\mathfrak{g}=\mathfrak{h} \oplus \mathfrak{m}, \operatorname{Ad}(H) \mathfrak{m} \subset \mathfrak{m}$. (If $G$ is connected and $M$ is simply connected then $H$ is connected, and the latter condition is equivalent to $[\mathfrak{h}, \mathfrak{m}] \subset \mathfrak{m}$.)

Let $(M, g)$ be a connected, simply connected and geodesically complete pseudo-Riemannian manifold, and suppose that $S$ is a homogeneous pseudo-Riemannian structure on $(M, g)$. We fix a point $o \in M$ and put $\mathfrak{m}=T_{o}(M)$. If $\widetilde{R}$ is the curvature tensor of the connection $\widetilde{\nabla}=\nabla-S$, we can consider the holonomy algebra $\tilde{\mathfrak{h}}$ of $\widetilde{\nabla}$ as the Lie subalgebra of antisymmetric endomorphisms of $\left(\mathfrak{m}, g_{o}\right)$ generated by the operators $\widetilde{R}_{Z W}$, where $Z, W \in \mathfrak{m}$. Then, according to the Ambrose-Singer construction [14, 15], a Lie bracket is defined in the vector space direct sum $\tilde{\mathfrak{g}}=\tilde{\mathfrak{h}} \oplus \mathfrak{m}$ by

$$
\begin{aligned}
& {[U, V]=U V-V U, \quad U, V \in \tilde{\mathfrak{h}},} \\
& {[U, Z]=U(Z), \quad U \in \tilde{\mathfrak{h}}, Z \in \mathfrak{m},} \\
& {[Z, W]=\widetilde{R}_{Z W}+S_{Z} W-S_{W} Z, \quad Z, W \in \mathfrak{m},}
\end{aligned}
$$

and we say that $(\tilde{\mathfrak{g}}, \tilde{\mathfrak{h}})$ is the reductive pair associated to the homogeneous pseudo-Riemannian structure $S$. The connected simply connected Lie group $\widetilde{G}$ whose Lie algebra is $\tilde{\mathfrak{g}}$ acts transitively on $M$ as a group of isometries and $M \equiv \widetilde{G} / \widetilde{H}$, where $\widetilde{H}$ is the connected Lie subgroup of $\widetilde{G}$ whose Lie algebra is $\tilde{\mathfrak{h}}$. The set $K$ of the elements of $\widetilde{G}$ which act trivially on $M$ is a discrete normal subgroup of $\widetilde{G}$, and the Lie group $G=\widetilde{G} / K$ acts transitively and effectively on $M$ as a group of isometries, with isotropy group $H=\widetilde{H} / K$. Then $M$ is (diffeomorphic to) the reductive homogeneous pseudo-Riemannian manifold $G / H$.

On the other hand, we consider (see e.g., Ref. [18], p. 134) the Einstein-Yang-Mills equations on a Lorentzian manifold $(M, g)$ :

$$
r-\frac{1}{2} s g+\Lambda g=\kappa T
$$

where $g$ denotes the metric tensor, $r$ the Ricci tensor and $s$ the scalar curvature of $g, \Lambda$ and $\kappa$ the cosmological and gravitational constants, respectively, and $T$ the stress-energy tensor of the gauge field with field strength $F$, given by

$$
T(X, Y)=g^{-1}\left(i_{X} F, i_{Y} F\right)-\frac{1}{4} g(X, Y)\|F\|^{2},
$$

$i$ denoting the interior product.

Let $\mathfrak{g}$ be the Lie algebra with generators $P, X, Y, Q$, and brackets

$$
[X, Y]=P, \quad[Q, X]=Y, \quad[Q, Y]=-X .
$$

The corresponding simply connected Lie group $G$ is called the oscillator group. 


\section{LORENTZIAN HOMOGENEOUS STRUCTURES, AND REDUCTIVE DECOMPOSITIONS}

Consider the biinvariant Lorentzian metric $g$ on the oscillator group $G$ given in the basis $\{P, X, Y, Q\}$, by

$$
g=\left(\begin{array}{llll} 
& & & 1 \\
& 1 & & \\
& & 1 & \\
1 & & &
\end{array}\right)
$$

Let $\{\eta, \alpha, \beta, \xi\}$ be the dual basis to $\{P, X, Y, Q\}$. The Levi-Civita connection $\nabla$ of $g$ is determined by $2 g\left(\nabla_{W} U, V\right)=g([W, U], V)-g([U, V], W)+g([V, W], U)$ for all $U, V, W \in \mathfrak{g}$. So, we obtain that $\nabla$ is given by

$$
\begin{aligned}
& \nabla_{W} P=0, \\
& \nabla_{W} X=-\frac{1}{2} \beta(W) P+\frac{1}{2} \xi(W) Y, \\
& \nabla_{W} Y=\frac{1}{2} \alpha(W) P-\frac{1}{2} \xi(W) X, \\
& \nabla_{W} Q=\frac{1}{2} \beta(W) X-\frac{1}{2} \alpha(W) Y,
\end{aligned}
$$

for every $W \in \mathfrak{g}$. The nonvanishing components of the curvature tensor field, for which we adopt the convention $R_{W U} V=\nabla_{[W, U]} V-\nabla_{W} \nabla_{U} V+\nabla_{U} \nabla_{W} V$, are given by

$$
R_{Q X} X=-\frac{1}{4} P, \quad R_{Q X} Q=\frac{1}{4} X, \quad R_{Q Y} Y=-\frac{1}{4} P, \quad R_{Q Y} Q=\frac{1}{4} Y .
$$

Thus, the only nonvanishing component of the Ricci tensor is $r(Q, Q)=\frac{1}{2}$ and the scalar curvature is $s=0$.

In Ref. [12] all the homogeneous Lorentzian structures on the general oscillator group are determined by solving the Ambrose-Singer equations (1). As a consequence, we have all the homogeneous Lorentzian structures on the 4-dimensional oscillator group.

Proposition. All the homogeneous Lorentzian structures on $(G, g)$ are given by

$$
S=\theta \otimes(\alpha \wedge \beta)+\rho \otimes(\alpha \wedge \xi)+\sigma \otimes(\beta \wedge \xi),
$$

where $\rho, \sigma$ and $\theta$ are left invariant 1 -forms on $G$ satisfying the conditions

$$
\begin{aligned}
& \widetilde{\nabla} \theta=0, \\
& \widetilde{\nabla} \rho=\sigma \wedge \theta-\frac{1}{2} \alpha \otimes \theta+\frac{1}{2} \xi \otimes \sigma, \\
& \widetilde{\nabla} \sigma=-\rho \wedge \theta-\frac{1}{2} \beta \otimes \theta-\frac{1}{2} \xi \otimes \rho,
\end{aligned}
$$

with $\widetilde{\nabla}=\nabla-S$.

Proof: The expression (6) follows from the equations $\widetilde{\nabla} g=0$ and $\widetilde{\nabla} R=0$ in (1). The conditions (7) are equivalent to the equation $\widetilde{\nabla} S=0$. (See Theorem 4.1 in Ref. [12].) 
By (6), we can write the following general expression for the homogeneous Lorentzian structures on $(G, g)$ :

$$
\begin{aligned}
S=( & \left.a_{1} \eta+a_{2} \alpha+a_{3} \beta+a_{4} \xi\right) \otimes(\alpha \wedge \beta) \\
& +\left(b_{1} \eta+b_{2} \alpha+b_{3} \beta+b_{4} \xi\right) \otimes(\alpha \wedge \xi) \\
& +\left(c_{1} \eta+c_{2} \alpha+c_{3} \beta+c_{4} \xi\right) \otimes(\beta \wedge \xi), \quad a_{1}, \ldots, c_{4} \in \mathbb{R} .
\end{aligned}
$$

The equations (7) gives us, after some long but straigthforward calculations, the relations to be satisfied by the coefficients. If $a_{1} \neq 0$ (we denote this case as $(i)$ ), we get the expression of $S$ in terms of $a_{1}, a_{2}, a_{3}, a_{4}$ :

$$
\begin{aligned}
S=\left(a_{1} \eta+a_{2} \alpha+a_{3} \beta+a_{4} \xi\right) \otimes(\alpha \wedge \beta) \\
+\left(-a_{3} \eta-\frac{a_{2} a_{3}}{a_{1}} \alpha-\left(\frac{a_{3}^{2}}{a_{1}}+\frac{1}{2}\right) \beta+\left(\frac{a_{3}}{2 a_{1}}-\frac{a_{3} a_{4}}{a_{1}}\right) \xi\right) \otimes(\alpha \wedge \xi) \\
+\left(a_{2} \eta+\left(\frac{a_{2}^{2}}{a_{1}}+\frac{1}{2}\right) \alpha+\frac{a_{2} a_{3}}{a_{1}} \beta+\left(\frac{a_{2} a_{4}}{a_{1}}-\frac{a_{2}}{2 a_{1}}\right) \xi\right) \otimes(\beta \wedge \xi) .
\end{aligned}
$$

If $a_{1}=0$, some tedious computations lead to another five cases, and the coefficients in the six cases $(i)$ to $(v i)$ are as follows

\begin{tabular}{l|llllll} 
& $(i)$ & $(i i)$ & $($ iii $)$ & $($ iv $)$ & $(v)$ & $(v i)$ \\
\hline$a_{1}$ & $\neq 0$ & 0 & 0 & 0 & 0 & 0 \\
$a_{2}$ & $\in \mathbb{R}$ & 0 & 0 & 0 & 0 & 0 \\
$a_{3}$ & $\in \mathbb{R}$ & 0 & 0 & 0 & 0 & 0 \\
$a_{4}$ & $\in \mathbb{R}$ & $\frac{1}{2}$ & $\frac{1}{2}$ & $\frac{1}{2}$ & $\neq \frac{1}{2}$ & $\neq \frac{1}{2}$ \\
$b_{1}$ & $-a_{3}$ & 0 & 0 & 0 & 0 & 0 \\
$b_{2}$ & $-\frac{a_{2} a_{3}}{a_{1}}$ & 0 & 0 & $\in \mathbb{R}$ & 0 & 0 \\
$b_{3}$ & $-\frac{a_{3}^{2}}{a_{1}}-\frac{1}{2}$ & $-\frac{1}{2}$ & $-\frac{1}{2}$ & $\neq-\frac{1}{2}$ & $-\frac{1}{2}$ & $-a_{4}$ \\
$b_{4}$ & $\frac{a_{3}}{2 a_{1}}-\frac{a_{3} a_{4}}{a_{1}}$ & 0 & $\in \mathbb{R}$ & $\in \mathbb{R}$ & $\in \mathbb{R}$ & 0 \\
$c_{1}$ & $a_{2}$ & 0 & 0 & 0 & 0 & 0 \\
$c_{2}$ & $\frac{a_{2}^{2}}{a_{1}}+\frac{1}{2}$ & $\neq \frac{1}{2}$ & $\frac{1}{2}$ & $\frac{1}{2}-\frac{2 b_{2}^{2}}{2 b_{3}+1}$ & $\frac{1}{2}$ & $a_{4}$ \\
$c_{3}$ & $\frac{a_{2} a_{3}}{a_{1}}$ & 0 & 0 & $-b_{2}$ & 0 & 0 \\
$c_{4}$ & $-\frac{a_{2}}{2 a_{1}}+\frac{a_{2} a_{4}}{a_{1}}$ & $\in \mathbb{R}$ & $\in \mathbb{R}$ & $-\frac{2 b_{2} b_{4}}{2 b_{3}+1}$ & $\in \mathbb{R}$ & 0
\end{tabular}

In the case $(i)$, making the change $x=a_{1} \neq 0, y=a_{2} / a_{1}, z=a_{3} / a_{1}, w=a_{4}-1 / 2$, we can 
write the family of homogeneous Lorentzian structures as

$$
\begin{aligned}
S_{(x, y, z, w)}=(x \eta+x y \alpha & \left.+x z \beta+\left(w+\frac{1}{2}\right) \xi\right) \otimes(\alpha \wedge \beta) \\
& -\left(x z \eta+x y z \alpha+\left(x z^{2}+\frac{1}{2}\right) \beta+z w \xi\right) \otimes(\alpha \wedge \xi) \\
& +\left(x y \eta+\left(x y^{2}+\frac{1}{2}\right) \alpha+x y z \beta+y w \xi\right) \otimes(\beta \wedge \xi), \\
& x, y, z, w \in \mathbb{R}, x \neq 0 .
\end{aligned}
$$

In the cases $(i i)$ to $(v i)$ one arrives (after some changes of notations for the coefficients) to the following families:

(ii)

$$
S_{(q, c)}=\frac{1}{2} \xi \otimes(\alpha \wedge \beta)-\frac{1}{2} \beta \otimes(\alpha \wedge \xi)+(q \alpha+c \xi) \otimes(\beta \wedge \xi), \quad q, c \in \mathbb{R}, q \neq \frac{1}{2} .
$$

$$
S_{(b, c)}=\frac{1}{2} \xi \otimes(\alpha \wedge \beta)+\left(-\frac{1}{2} \beta+b \xi\right) \otimes(\alpha \wedge \xi)+\left(\frac{1}{2} \alpha+c \xi\right) \otimes(\beta \wedge \xi), b, c \in \mathbb{R} .
$$

$$
\begin{aligned}
S_{(k, t, b)}=\frac{1}{2} \xi \otimes(\alpha \wedge \beta) & +\left(k \alpha+\left(t-\frac{1}{2}\right) \beta+b \xi\right) \otimes(\alpha \wedge \xi) \\
& +\left(\left(\frac{1}{2}-\left(k^{2} / t\right)\right) \alpha-k \beta-(k b / t) \xi\right) \otimes(\beta \wedge \xi), \quad k, t, b \in \mathbb{R}, t \neq 0 .
\end{aligned}
$$

$(v)$

$$
S_{(a, b, c)}=a \xi \otimes(\alpha \wedge \beta)+\left(-\frac{1}{2} \beta+b \xi\right) \otimes(\alpha \wedge \xi)+\left(\frac{1}{2} \alpha+c \xi\right) \otimes(\beta \wedge \xi), \quad
$$

$$
S_{a}=a(\xi \otimes(\alpha \wedge \beta)-\beta \otimes(\alpha \wedge \xi)+\alpha \otimes(\beta \wedge \xi)), \quad a \in \mathbb{R}, a \neq \frac{1}{2} .
$$

Next, we determine $\widetilde{\nabla}=\nabla-S$. We however omit the expressions of $\widetilde{\nabla}$ for the sake of brevity. Then, in each case we calculate the curvature $\widetilde{R}$, and so we obtain generators of the holonomy algebra $\tilde{\mathfrak{h}}$ of $\widetilde{\nabla}$, which will be expressed in terms of the basis $\{P, X, Y, Q\}$ of $\mathfrak{g}$.

In the case $(i)$, we have that the only not always null curvature operators are $\widetilde{R}_{X Y}=w U$, $\widetilde{R}_{Q X}=x z U$ and $\widetilde{R}_{Q Y}=-x y U$, where

$$
U=\left(\begin{array}{rrrr}
0 & z & -y & 0 \\
0 & 0 & 1 & -z \\
0 & -1 & 0 & y \\
0 & 0 & 0 & 0
\end{array}\right)
$$

If $y=z=w=0$ then $\widetilde{R}=0$, but if either $y, z$, or $w$ is not zero then the holonomy algebra $\tilde{\mathfrak{h}}$ is 1-dimensional and generated by $U$.

In the case $(i i)$, the holonomy algebra $\tilde{\mathfrak{h}}$ is generated by the vector

$$
V=\left(\begin{array}{rrrr}
0 & 0 & 1 & 0 \\
0 & 0 & 0 & 0 \\
0 & 0 & 0 & -1 \\
0 & 0 & 0 & 0
\end{array}\right)
$$


and the only nonvanishing curvature operator is $\widetilde{R}_{Q Y}=\left(q-\frac{1}{2}\right) V$.

In the case $($ iii $)$ (and $(v)$ ) we deduce $\widetilde{R}=0$.

In the case $(i v)$, the holonomy algebra $\tilde{\mathfrak{h}}$ is generated by

$$
U=\left(\begin{array}{rrrr}
0 & -t & k & 0 \\
0 & 0 & 0 & t \\
0 & 0 & 0 & -k \\
0 & 0 & 0 & 0
\end{array}\right)
$$

and the curvature of $\widetilde{\nabla}$ is determined by $\widetilde{R}_{Q X}=U$ and $\widetilde{R}_{Q Y}=-(k / t) U$.

In the case $(v i)$, the holonomy algebra $\tilde{h}$ has two generators

$$
U=\left(\begin{array}{rrrr}
0 & 1 & 0 & 0 \\
0 & 0 & 0 & -1 \\
0 & 0 & 0 & 0 \\
0 & 0 & 0 & 0
\end{array}\right), \quad V=\left(\begin{array}{rrrr}
0 & 0 & 1 & 0 \\
0 & 0 & 0 & 0 \\
0 & 0 & 0 & -1 \\
0 & 0 & 0 & 0
\end{array}\right)
$$

and the curvature is determined by the operators $\widetilde{R}_{Q X}=\left(a^{2}-\frac{1}{4}\right) U, \widetilde{R}_{Q Y}=\left(a^{2}-\frac{1}{4}\right) V$. If $a=-\frac{1}{2}$ then $\widetilde{R}=0$ and if $a \neq-\frac{1}{2}$, then $\tilde{\mathfrak{h}}$ is 2 -dimensional and abelian.

Through the usual identification of $\mathfrak{g}$ with the tangent space at the identity of $G$, we consider $\mathfrak{m}=\mathfrak{g}$ and use (2) in order to obtain the Lie bracket of $\tilde{\mathfrak{g}}=\tilde{\mathfrak{h}} \oplus \mathfrak{g}$ in each case. In particular, we will have the reductive pair $(\tilde{\mathfrak{g}}, \tilde{\mathfrak{h}})$ associated to each homogeneous structure.

We next explicitly give those brackets. Some cases are broken in two subcases according to whether the dimension of the holonomy algebra depends on the particular values of the coefficients in the corresponding homogeneous structure.

Case $\left(i_{1}\right) \quad(x, y, z, w \in \mathbb{R} ; x \neq 0 ; y, z, w$ not all null). The reductive decomposition associated to $S_{(x, y, z, w)}$ is with not always null brackets

$$
\begin{gathered}
{[U, X]=z P-Y, \quad[U, Y]=-y P+X, \quad[U, Q]=-z X+y Y,} \\
{[P, X]=-x z P+x Y, \quad[P, Y]=x y P-x X, \quad[P, Q]=x z X-x y Y,} \\
{[X, Y]=w U+\left(x\left(y^{2}+z^{2}\right)+1\right) P-x y X-x z Y,} \\
{[Q, X]=x z U-z w P-x y z X+\left(w+x y^{2}+1\right) Y,} \\
{[Q, Y]=-x y U+y w P-\left(w+x z^{2}+1\right) X+x y z Y .}
\end{gathered}
$$

Case $\left(i_{2}\right) \quad(x \neq 0 ; y=z=w=0)$. The reductive decomposition associated to $S_{(x, 0,0,0)}$ is with nonvanishing brackets

$$
[P, X]=x Y, \quad[P, Y]=-x X, \quad[X, Y]=P, \quad[Q, X]=Y, \quad[Q, Y]=-X .
$$

Case $(i i) \quad\left(q, c \in \mathbb{R} ; q \neq \frac{1}{2}\right)$. The reductive decomposition associated to $S_{(q, c)}$ is $\tilde{\mathfrak{g}}_{(q, c)}=$ $\tilde{\mathfrak{h}} \oplus \mathfrak{g}=\langle\{V, P, X, Y, Q\}\rangle$, with nonvanishing brackets

$$
\begin{aligned}
& {[V, Y]=P, \quad[V, Q]=-Y,} \\
& {[Q, X]=\left(q+\frac{1}{2}\right) Y, \quad[Q, Y]=\left(q-\frac{1}{2}\right) V+c P-X \text {. }} \\
& {[X, Y]=\left(q+\frac{1}{2}\right) P}
\end{aligned}
$$


Case $(i i i) \quad(b, c \in \mathbb{R})$. The reductive decomposition associated to $S_{(b, c)}$ is $\tilde{\mathfrak{g}}_{(b, c)}=0 \oplus \mathfrak{g}=$ $\langle\{P, X, Y, Q\}\rangle$, with nonvanishing brackets

$$
[X, Y]=P, \quad[Q, X]=b P+Y, \quad[Q, Y]=c P-X
$$

In this case, one obtains the Lie algebra of the oscillator group. In fact, putting $\widetilde{X}=X-c P$, $\widetilde{Y}=Y+b P$, we have $[\widetilde{X}, \widetilde{Y}]=P,[Q, \widetilde{X}]=\widetilde{Y},[Q, \widetilde{Y}]=-\widetilde{X}$.

Case $(i v) \quad(k, t, b \in \mathbb{R} ; t \neq 0)$. The reductive decomposition associated to $S_{(k, t, b)}$ is $\tilde{\mathfrak{g}}_{(k, t, b)}=\tilde{\mathfrak{h}} \oplus \mathfrak{g}=\langle\{U, P, X, Y, Q\}\rangle$, with Lie brackets given by

$$
\begin{aligned}
& {[U, X]=-t P, \quad[U, Y]=k P, \quad[U, Q]=t X-k Y, \quad[X, Y]=\left(1-\left(k^{2}+t^{2}\right) / t\right) P,} \\
& {[Q, X]=U+b P+k X+\left(1-\frac{k^{2}}{t}\right) Y, \quad[Q, Y]=-\frac{k}{t} U-\frac{k b}{t} P+(t-1) X-k Y .}
\end{aligned}
$$

Case $(v) \quad\left(a, b, c \in \mathbb{R} ; a \neq \frac{1}{2}\right)$. The reductive decomposition associated to $S_{(a, b, c)}$ is $\tilde{\mathfrak{g}}_{(a, b, c)}=0 \oplus \mathfrak{g}=\langle\{P, X, Y, Q\}\rangle$, with nonvanishing brackets

$$
[X, Y]=P, \quad[Q, X]=b P+\left(a+\frac{1}{2}\right) Y, \quad[Q, Y]=c P-\left(a+\frac{1}{2}\right) X
$$

Case $\left(v i_{1}\right) \quad\left(a \in \mathbb{R} ; a \neq \frac{1}{2},-\frac{1}{2}\right)$. The reductive decomposition associated to $S_{a}$ is $\tilde{\mathfrak{g}}_{a}=$ $\tilde{\mathfrak{h}} \oplus \mathfrak{g}=\langle\{U, V, P, X, Y, Q\}\rangle$, with nonvanishing brackets

$$
\begin{aligned}
& {[U, X]=P, \quad[U, Q]=-X, \quad[V, Y]=P, \quad[V, Q]=-Y, \quad[X, Y]=2 a P} \\
& {[Q, X]=\left(a^{2}-\frac{1}{4}\right) U+2 a Y, \quad[Q, Y]=\left(a^{2}-\frac{1}{4}\right) V-2 a X}
\end{aligned}
$$

In particular, if $a=0$, one has $S=0$, and the associated reductive pair $(\tilde{\mathfrak{g}}, \tilde{\mathfrak{h}})$ is a symmetric pair, which defines the oscillator group as a symmetric Lorentzian space.

Case $\left(v i_{2}\right) \quad\left(a=-\frac{1}{2}\right)$. The reductive decomposition associated to $S_{-\frac{1}{2}}$ is $\tilde{\mathfrak{g}}_{-\frac{1}{2}}=0 \oplus \mathfrak{g}=$ $\langle\{P, X, Y, Q\}\rangle$, with nonvanishing brackets

$$
[X, Y]=-P, \quad[Q, X]=-Y, \quad[Q, Y]=X,
$$

which also gives the Lie algebra of the oscillator group.

\section{CURRENTS, AND EINSTEIN-YANG-MILLS EQUATIONS}

We consider the connection form $\omega$ on $\tilde{\mathfrak{g}}$, which is the $\tilde{\mathfrak{h}}$-component of the canonical 1-form on $\tilde{\mathfrak{g}}$, and we obtain, in each case, the curvature form $F=d \omega$ and then $\star F$, where $\star$ denotes 
the Hodge operator with respect to the Lorentzian metric $g$. The following table provides the values of $F$ and $\star F$ for the cases with non-trivial holonomy.

$$
\begin{array}{r|cc} 
& F=d \omega & \star F \\
\hline\left(i_{1}\right) & (-w \alpha \wedge \beta+x z \alpha \wedge \xi-x y \beta \wedge \xi) \otimes U & -(w \eta \wedge \xi+x z \eta \wedge \beta+x y \eta \wedge \alpha) \otimes U \\
(i i) & \left(q-\frac{1}{2}\right)(\beta \wedge \xi) \otimes V & \left(q-\frac{1}{2}\right)(\eta \wedge \alpha) \otimes V \\
(i v) & (\alpha \wedge \xi-(k / t) \beta \wedge \xi) \otimes U & -(\eta \wedge \beta+(k / t) \eta \wedge \alpha) \otimes U \\
\left(v i_{1}\right) & \left(a^{2}-\frac{1}{4}\right)((\alpha \wedge \xi) \otimes U+(\beta \wedge \xi) \otimes V) & \left(a^{2}-\frac{1}{4}\right)(-(\eta \wedge \beta) \otimes U+(\eta \wedge \alpha) \otimes V)
\end{array}
$$

Moreover, as the second Yang-Mills equation is $D \star F=J$, where $J$ denotes the current, applying the usual formula [19] one obtains for all the cases

$$
\begin{aligned}
J & =D \star F \\
& =d \star F+\omega \wedge \star F-\star F \wedge \omega \\
& =d \star F .
\end{aligned}
$$

Thus, the currents are given, respectively, by

$$
\begin{array}{r|l} 
& J \\
\hline\left(i_{1}\right) & (x z \eta \wedge \alpha \wedge \xi-x y \eta \wedge \beta \wedge \xi+w \alpha \wedge \beta \wedge \xi) \otimes U \\
(i i) & \left(q-\frac{1}{2}\right)(\eta \wedge \beta \wedge \xi+c \alpha \wedge \beta \wedge \xi) \otimes V \\
(i v) & \left(\eta \wedge \alpha \wedge \xi-(k / t) \eta \wedge \beta \wedge \xi+b\left(1+(k / t)^{2}\right) \alpha \wedge \beta \wedge \xi\right) \otimes U \\
\left(v i_{1}\right) & 2 a\left(a^{2}-\frac{1}{4}\right)((\eta \wedge \alpha \wedge \xi) \otimes U+(\eta \wedge \beta \wedge \xi) \otimes V)
\end{array}
$$

On the other hand, we consider the metric on $\tilde{\mathfrak{g}}$ which is the direct product of the metric $g_{0}$ on $\mathfrak{g}$ and the metric on $\tilde{\mathfrak{h}}$ for which the basic vectors $(U$ and/or $V$ ) are unitary.

Now we have $\|F\|^{2}=2 w^{2}$ in the case $\left(i_{1}\right)$ and $\|F\|^{2}=0$ in the remaining cases. We next compute the stress-energy tensor $T$, given by (4), and we express it in terms of the basis $\{P, X, Y, Q\}$ of $\mathfrak{g}$. In the case $\left(i_{1}\right)$ we have

$$
T=\left(\begin{array}{cccc}
0 & 0 & 0 & -\frac{1}{2} w^{2} \\
0 & \frac{1}{2} w^{2} & 0 & -x y w \\
0 & 0 & \frac{1}{2} w^{2} & -x z w \\
-\frac{1}{2} w^{2} & -x y w & -x z w & x^{2}\left(y^{2}+z^{2}\right)
\end{array}\right) .
$$

In the cases $(i i),(i v)$ and $\left(v i_{1}\right)$, the only nonvanishing component is $T(Q, Q)$, which is, respectively, $\left(q-\frac{1}{2}\right)^{2}, 1+(k / t)^{2}$ and $2\left(a^{2}-\frac{1}{4}\right)^{2}$. 
It is then a matter of calculation to see that the field equations (3) are satisfied in the cases $(i i),(i v)$ and $(v i)$, and in the case $\left(i_{1}\right)$ if $w=0$ and $y^{2}+z^{2} \neq 0$, and to obtain the corresponding cosmological and gravitational constants.

Theorem. The Lorentzian reductive pairs $(\tilde{\mathfrak{g}}, \tilde{\mathfrak{h}})$ determined by the homogeneous Lorentzian structures on the 4-dimensional oscillator group, in the cases $\left(i_{1}\right)$ (with $w=0$ and $\left.y^{2}+z^{2} \neq 0\right),(i i),(i v)$ and $\left(v i_{1}\right)$ above, are solutions of the Einstein-Yang-Mills equations for an electromagnetic gauge field with cosmological constant $\Lambda=0$, and gravitational constant $\kappa$ given, respectively, by

$$
\frac{1}{2 x^{2}\left(y^{2}+z^{2}\right)}, \quad \frac{1}{2\left(q-\frac{1}{2}\right)^{2}}, \quad \frac{t^{2}}{2\left(k^{2}+t^{2}\right)}, \quad \frac{1}{4\left(a^{2}-\frac{1}{4}\right)^{2}} .
$$

The gauge algebra $\tilde{\mathfrak{h}}$ is abelian and 2-dimensional in the last case and 1-dimensional in the other three cases. The currents are given in the table following (8). They are nonvanishing except in the case $\left(v i_{1}\right)$ for $a=0$.

If $a=0$ in the case $\left(v i_{1}\right)$, the associated symmetric pair $(\tilde{\mathfrak{g}}, \tilde{\mathfrak{h}})$ appears as a solution of the Einstein-Yang-Mills equations for a sourceless gauge field with cosmological constant $\Lambda=0$ and gravitational constant $\kappa=4$ (see Levichev [5]).

\section{ACKNOWLEDGEMENTS}

This work has been partially supported by DGES (Spain) under Project PB95-0124, and by Xunta de Galiza, under Project XUGA 20703B98.

\section{References}

[1] R. F. Streater, "The representations of the oscillator group", Comm. Math. Phys. 4, 217-236 (1967).

[2] T. Nomura, "The Paley-Wiener theorem for the oscillator group", J. Math. Kyoto Univ. 22, 71-96 (1982/83).

[3] A. Medina, "Groupes de Lie munis de métriques biinvariantes", Tôhoku Math. J. 37, 405-421 (1985).

[4] A. Medina and P. Revoy, "Les groupes oscillateurs et leurs réseaux", Manuscripta Math. 52, 81-95 (1985).

[5] A. V. Levichev, "Several symmetric spaces of general relativity theory as solutions of EinsteinYang-Mills equations", in Group theoretical methods in physics, Proc. 3rd Intern. Sem., Yurmala, May 1985, vol. 1, (Nauka, Moscow, 1986), pp. 145-150 (in Russian).

[6] A. V. Levichev, "Chronogeometry of an electromagnetic wave given by a biinvariant metric on the oscillator group", Siberian Math. J. 27, 237-245 (1986).

[7] D. Müller and F. Ricci, "On the Laplace-Beltrami operator on the oscillator group", J. Reine Angew. Math. 390, 193-207 (1988). 
[8] A. V. Levichev, "Methods of investigation of the causal structure of homogeneous Lorentz manifolds", Siberian Math. J. 31, 395-408 (1990).

[9] N. Dörr, "A note on the oscillator group", Seminar Sophus Lie 2, 31-38 (1991).

[10] O. S. Svidersky, "Oscillator parallelization of the scalar induced bundle", Siberian Mat. J. 36, 965-972 (1995).

[11] O. S. Svidersky, "Decomposition of a vector field in a parallelized induced bundle", Proc. Amer. Math. Soc. 125, 2485-2491 (1997).

[12] P. M. Gadea and J. A. Oubiña, "Homogeneous Lorentzian structures on the oscillator groups", to appear in Arch. Math. (Basel).

[13] É. Cartan, "Sur une classe remarquable d'espaces de Riemann", Bull. Soc. Math. France 54 214-264 (1926).

[14] W. Ambrose and I. M. Singer, "On homogeneous Riemannian manifolds", Duke Math. J. 25, 647-669 (1958).

[15] F. Tricerri and L. Vanhecke, Homogeneous Structures on Riemannian Manifolds, London Math. Soc. Lect. Notes Ser. 83, (Cambridge Univ. Press, Cambridge, U.K., 1983).

[16] P. M. Gadea and J. A. Oubiña, "Homogeneous pseudo-Riemannian structures and homogeneous almost para-Hermitian structures", Houston J. Math. 18, 449-465 (1992).

[17] P. M. Gadea and J. A. Oubiña, "Reductive homogeneous pseudo-Riemannian manifolds", Monatsh. Math. 124, 17-34 (1997).

[18] D. Bleecker, Gauge Theories and Variational Principles, Addison-Wesley, Reading, Massachusetts, 1981.

[19] T. Eguchi, P. B. Gilkey and A. J. Hanson, "Gravitation, gauge theories and differential geometry", Phys. Rep. (Review sect. of Phys. Lett.) 66, n. 6, 213-393 (1980). 